\title{
Criminologie
}

\section{La victimisation : un aspect marquant de l'expérience des jeunes filles dans les gangs}

\section{Michèle Fournier, Marie-Marthe Cousineau et Sylvie Hamel}

Volume 37, numéro 1, printemps 2004

Criminologie : discipline et institutionnalisation. Trois exemples francophones

URI : https://id.erudit.org/iderudit/008721ar

DOI : https://doi.org/10.7202/008721ar

Aller au sommaire du numéro

\section{Éditeur(s)}

Les Presses de l'Université de Montréal

ISSN

0316-0041 (imprimé)

1492-1367 (numérique)

Découvrir la revue

Citer cet article

Fournier, M., Cousineau, M.-M. \& Hamel, S. (2004). La victimisation : un aspect marquant de l'expérience des jeunes filles dans les gangs. Criminologie, 37(1), 149-166. https://doi.org/10.7202/008721ar
Résumé de l'article

L'intérêt des chercheurs pour le phénomène des gangs de rue n'est pas nouveau, comme en font foi les travaux de Trasher publiés dès 1927, ou ceux de Cohen, qui datent de 1955. Au fil des ans, de nombreuses recherches ont été menées sur le sujet et ont permis d'élargir, de façon considérable, les connaissances s'y rattachant. Pourtant, les écrits scientifiques portant spécifiquement sur la présence et le vécu des filles au sein des gangs se font rares, particulièrement au Québec. Tiré d'une recherche qualitative portant sur le cheminement et le vécu des adolescentes montréalaises affiliées à un gang, cet article s'intéresse à un aspect particulier de l'expérience qu'y vivent les filles, celui de la victimisation. Par le biais de récits d'expériences, treize adolescentes ont raconté ce qu'elles ont connu des gangs pendant la période où elles y ont été affiliées. Leurs propos permettent d'apprendre que les jeunes filles qui se joignent à un gang de rue peuvent être victimes de diverses formes de violence au sein de celui-ci : agressions physiques, contrôle, violence amoureuse, isolement, victimisation sexuelle et menaces. De telles expériences présentent un défi pour les personnes oeuvrant auprès de ces jeunes filles, d'autant plus qu'il semble y avoir une certaine distance entre ce qu'elles considèrent comme des éléments victimisants et ce que les jeunes filles estiment être de la victimisation.
Ce document est protégé par la loi sur le droit d'auteur. L'utilisation des services d'Érudit (y compris la reproduction) est assujettie à sa politique d'utilisation que vous pouvez consulter en ligne.

https://apropos.erudit.org/fr/usagers/politique-dutilisation/ 


\title{
La victimisation : un aspect marquant de l'expérience des jeunes filles dans les gangs
}

\author{
Michèle Fournier \\ Candidate au doctorat \\ École de criminologie \\ Université de Montréal \\ michele.fournier@questzones.ca \\ Marie-Marthe Cousineau \\ Professeure, École de criminologie \\ Chercheure, Centre international de criminologie comparée \\ Université de Montréal \\ cousinem@cicc.ca \\ Sylvie Hamel \\ Chercheure \\ Institut de recherche pour \\ le développement social de jeunes \\ shamel@montreal.centresjeunesse.qc.ca
}

RÉSUMÉ • L'intérêt des chercheurs pour le phénomène des gangs de rue n'est pas nouveau, comme en font foi les travaux de Trasher publiés dès 1927, ou ceux de Cohen, qui datent de 1955. Au fil des ans, de nombreuses recherches ont été menées sur le sujet et ont permis d'élargir, de façon considérable, les connaissances s'y rattachant. Pourtant, les écrits scientifiques portant spécifiquement sur la présence et le vécu des filles au sein des gangs se font rares, particulièrement au Québec. Tiré d'une recherche qualitative portant sur le cheminement et le vécu des adolescentes montréalaises affiliées à un gang, cet article s'intéresse à un aspect particulier de l'expérience qu'y vivent les filles, celui de la victimisation. Par le biais de récits d'expériences, treize adolescentes ont raconté ce qu'elles ont connu des gangs pendant la période où elles y ont été affiliées. Leurs propos permettent d'apprendre que les jeunes filles qui se joignent à un gang de rue peuvent être victimes de diverses formes de violence au sein de celui-ci: agressions physiques, contrôle, violence amoureuse, isolement, victimisation sexuelle et menaces. De telles expériences présentent un défi pour les personnes œuvrant auprès de ces jeunes filles, d'autant plus qu'il semble y avoir une certaine distance entre ce qu'elles considèrent comme des éléments victimisants et ce que les jeunes filles estiment être de la victimisation. 
ABSTRACT - Scholarly interest in the activities of street gangs is not new, as attested by early works by Trasher (1927) and Cohen (1955). Yet, although much has been explored on gangs throughout the decades since these studies, research relating specifically to the presence and actual experiences of girls within gangs remain rare. The Quebec context is not an exception in this general lapse of knowledge. This article is based on the results from a qualitative research on the development and experiences of young girls affiliated to a gang within the Montreal context. Particular interest is on the violent victimization that these girls experienced. Such victimization within street gang contexts may come in many forms: threats, physical aggression, control, isolation, sexual victimization, violence in a boyfriend/girlfriend relationship. These types of experiences bring a new challenge for those working with young girls, all the more so since it is likely that there seems to be a discrepancy on what constitutes victimization between counsellors and teenage girls.

Les chercheurs s'intéressant à la question s'entendent généralement pour dire que les études ayant porté spécifiquement sur les filles affiliées aux gangs ${ }^{1}$ ou membres de ceux-ci sont encore peu nombreuses (Joe et Chesney-Lind, 1995 ; Fagan, 1996; Shelden et al., 1996; Covey et al., 1997; Esbensen et Deschenes, 1998). Molidor (1996), réalisant une recension des écrits sur le sujet, fait d'ailleurs une critique sévère de la recherche concernant les filles et les gangs, affirmant que le matériel est pour le moins incomplet et dans une large mesure dépassé, plusieurs des recherches qu'il a répertoriées ayant été réalisées dans les années 1970 ou même avant. Chesney-Lind et al. (1996) constatent toutefois que, depuis le début des années 1990, on a vu naitre un intérêt particulier pour les filles délinquantes engagées dans des délits traditionnellement masculins, en particulier en contexte de gangs (Curry, 1998; Esbensen et al., 1999). Pour sa part, Miller (1998) signale que les recherches ont essentiellement documenté l'impact des gangs sur l'implication criminelle des filles, mais que peu d'attention a été accordée à son pendant, la victimisation.

De fait, plusieurs chercheurs s'accordent pour dire que les filles qui cherchent à s'associer à un gang de rue ont souvent été victimisées avant de s'y joindre (Klein, 1995; Molidor, 1996; Esbensen et al. 1999). La violence familiale et sexuelle est ainsi un thème récurrent dans l'histoire des filles membres de gangs (Campbell, 1984; Harris, 1988; Joe et Chesney-Lind, 1995).

1. Dans le cadre de cet article, les termes gang et bande seront tous deux employés, le même sens leur étant attribué. 
D'autres études ont montré que les occasions de victimisation ne se limitent pas à la période précédant l'affiliation aux gangs, mais qu'une telle association entraînerait, elle-même, des épisodes de victimisation. Ainsi, des jeunes filles seraient victimisées dès leurs premiers contacts avec la bande, lors de l'initiation qui prendrait souvent la forme d'une agression sexuelle (Campbell, 1984; Molidor, 1996; Joe Laidler et Hunt, 1997; Miller, 1998). La victimisation se répèterait, dans bien des cas, au-delà de l'initiation, tant et aussi longtemps que durerait l'association au gang. Il arriverait ainsi que les filles soient giflées, frappées à coups de pied, étranglées, qu'elles soient menacées avec des armes ou encore battues sévèrement (Molidor, 1996). Les abus sexuels seraient également présents au sein même du gang, les adolescentes devant parfois se prêter à des relations sexuelles sur demande (Molidor, 1996; Huff, 1997; Joe Laidler et Hunt, 1997).

Les agressions au sein du gang se feraient aussi sentir lorsque les filles enfreignent les lois et règlements qui prévalent dans le groupe. En agissant de la sorte, elles s'exposeraient à des punitions physiques (Miller, 1998). Joe Laidler et Hunt (1997) ajoutent que les garçons du gang, même s'il s'agit des amoureux, peuvent se montrer contrôlants, abusifs, violents verbalement, physiquement et sexuellement.

Lauritsen et al. (1991) rapportent, quant à eux, que l'implication des adolescentes dans un style de vie déviant, se traduisant notamment dans l'affiliation à un gang, augmente fortement le risque de victimisation ${ }^{2}$. Dans le cas des gangs, les possibilités de confrontation avec des membres d'une bande adverse augmentent encore les risques d'agression.

Molidor (1996) rapporte enfin que la peur, et même une certaine forme de paranoïa développée par les filles, sont dénoncées par celles-ci comme étant les aspects les plus négatifs de leur affiliation au gang. Les filles rapportent qu'elles ne savent jamais quand elles peuvent être frappées ou attaquées non seulement par des membres de bandes adverses, mais au sein même de leur propre gang.

Ces données proviennent essentiellement d'études américaines. Nous avons voulu savoir dans quelle mesure elles pouvaient s'appliquer à l'affiliation des filles aux gangs de rue en contexte montréalais ${ }^{3}$.

2. Les garçons sont aussi exposés à diverses formes de victimisation dans ce contexte. Notre propos se limite toutefois ici à l'expérience des filles.

3. Le phénomène des gangs de rue, bien qu'il ait tendance à s'étendre aux banlieues, demeure essentiellement encore un phénomène de grandes villes. Au Québec, c'est dans la grande région de Montréal que les gangs de rues sont surtout réputés être actifs (Cousineau et al., 2003b). 


\section{Notre étude}

Issu des données d'une étude qualitative plus large (Fournier 2003, sous la direction de M.-M. Cousineau) portant sur le cheminement et les expériences vécues par les jeunes filles affiliées aux gangs de rue à Montréal, le présent article traite plus spécialement de la victimisation subie par ces jeunes filles du fait de leur affiliation à de tels groupes.

Cette victimisation peut prendre la forme d'agressions physiques, de violence amoureuse et de contrôle, d'isolement, d'abus ou de violence sexuelle et, finalement, de menaces pouvant être proférées à l'endroit de celles qui manifestent le désir de quitter le gang.

Dans le cadre de l'étude qui donne lieu au présent article, un gang de rue est défini comme étant un «regroupement d'adolescents et/ou de jeunes adultes, ayant un certain niveau d'organisation et qui commet des délits, notamment des délits de violence». Sachant que la définition de cette notion fait l'objet de nombreux débats et discussions, il a été convenu de considérer celle-ci d'une manière assez large, tout en prenant soin de faire la distinction entre les gangs de rue et les groupes d'amis auxquels se joignent la plupart des adolescents.

\section{Méthodologie}

\section{Stratégies d'échantillonnage}

La première préoccupation a été de distinguer les filles affiliées aux gangs de rue de celles faisant partie de groupes d'amis ayant pu, à l'occasion, verser dans la délinquance. Cette distinction est d'autant plus importante que les regroupements entre copains sont reconnus comme étant tout à fait sains pendant la période de l'adolescence, dans la mesure où ils ne sont pas déviants ou criminalisés (Fréchette et LeBlanc, 1997). Une attention particulière a donc été accordée à la violence attribuée aux gangs de rue, de même qu'à l'organisation de ceux-ci, spécialement en ce qui a trait à la hiérarchie que l'on y retrouve. Concernant le degré d'affiliation des filles aux gangs ou leur statut en regard du gang fréquenté, aucun critère de sélection n'a été retenu. Cette décision s'appuie sur le fait que, sans en devenir officiellement membres, les filles peuvent parfois fréquenter ou côtoyer de tels regroupements et en vivre l'expérience.

C'est par la technique du tri expertisé, qui consiste à faire appel à des spécialistes (Angers, 1992), que les jeunes filles rencontrées ont pu être 
identifiées et approchées. Pour ce faire, nous avons pris contact avec des intervenants de confiance amenés à travailler auprès des adolescentes membres ou affiliées à un gang de rue, au sens de la définition retenue, et qui étaient ainsi en mesure de confirmer que les jeunes filles ont vécu une telle expérience.

\section{Les entretiens}

L'importance de comprendre la façon dont les adolescentes interprètent leur propre situation en accordant une place centrale à leurs points de vue ayant été révélée par la recension des écrits, l'approche qualitative est apparue comme la plus appropriée pour répondre à ce besoin. Le récit d'expérience, qui peut être défini comme une entrevue de recherche visant à faire parler l'interviewé d'une période précise de son existence au cours de laquelle il a vécu une expérience particulière, a été privilégié. Une telle approche s'avérait particulièrement pertinente dans le cadre de cette étude, étant donné qu'elle visait à rendre compte du cheminement et de l'expérience des jeunes filles à une période particulière de leur vie, soit à partir du moment où elles ont eu leurs premiers contacts avec un gang de rue jusqu'à ce qu'elles quittent celui-ci, le cas échéant. La consigne marquant le départ des entrevues a pris la forme suivante:

J'aimerais que tu me racontes ton expérience à partir du moment où tu as eu tes premiers contacts avec un gang de rue.

Lors des entretiens, d'une durée allant d'une heure et quinze minutes à deux heures, des sous-consignes ont été lancées lorsque les jeunes filles n'avaient pas abordé certains thèmes d'elles-mêmes. Ces sous-consignes concernaient le processus d'affiliation au gang, l'expérience qui y était vécue, les relations entretenues à l'extérieur du gang, ainsi que le processus de désaffiliation. Des relances ont également été introduites, afin d'amener les participantes à approfondir leur propos et à préciser leur expérience.

Mentionnons, d'entrée de jeu, qu'il a été difficile de trouver des jeunes filles prêtes à parler de leur expérience avec les gangs de rue. La peur d'être reconnues et la crainte de représailles de la part des membres de la bande ont semblé faire hésiter plus d'une. Celles-ci ont dû être rassurées fréquemment quant aux visées de la recherche et au caractère confidentiel et anonyme de celle-ci. D'ailleurs, il s'est rapidement avéré que les adolescentes rencontrées avaient généralement plus de facilité à parler des autres jeunes filles que d'elles-mêmes. Il n'en demeure pas moins 
que si elles ne parlaient pas toujours en termes d'expérience personnelle, le récit qu'elles ont livré a tout de même contribué à lever le voile de manière significative sur le vécu des adolescentes affiliées à un gang.

\section{Les participantes}

Au moment de l'entrevue, l'âge des treize jeunes filles rencontrées variait de quatorze à vingt-quatre ans (moyenne: 15,9 ans). La limite inférieure est imposée par les restrictions concernant le consentement des parents, exigé pour que le témoignage soit possible. La limite supérieure répond à un souci d'hétérogénéité des expériences prises en compte. En effet, comme le phénomène des gangs de rue se révèle passablement changeant (Hamel et al., 1998), l'expérience des jeunes filles interviewées se devait d'être assez récente pour bien rendre compte de ce qui était vécu par les adolescentes fréquentant des gangs au moment où se tenait l'étude.

Lorsqu'elles ont été rencontrées, douze des adolescentes étaient placées en centre de réadaptation, sous le couvert de la Loi sur la protection de la jeunesse $\left(\mathrm{LPJ}^{4}\right)$. Parmi ces dernières, sept n'en étaient pas à leur premier contact avec les diverses formes de prise en charge institutionnelle, puisqu'elles avaient déjà été placées soit en famille d'accueil, soit en foyer de groupe, soit en centre de réadaptation. Lors des entretiens, neuf adolescentes affirmaient avoir quitté le gang qu'elles fréquentaient, alors que quatre disaient y être encore affiliées. Toutefois, parmi celles qui soutenaient avoir quitté le gang, huit confiaient avoir encore des contacts occasionnels avec les membres de celui-ci.

\section{Les stratégies d'analyse}

Les entrevues ont été intégralement retranscrites au fur et à mesure qu'elles ont été réalisées. Par la suite, deux types d'analyse ont été menés. D'abord, des analyses verticales de chaque entretien pris pour lui-même ont permis d'identifier les thèmes et les sous-thèmes qui en sont ressortis et d'approfondir, au fur et à mesure de l'avancement de la collecte de données, certaines dimensions abordées lors des premières entrevues, qui n'étaient pas apparues dans la recension des écrits. Dans un deuxième

4. Au Québec, les adolescent(e)s peuvent être pris(es) en charge par le réseau des Centres jeunesse en vertu de deux lois: la Loi sur la protection de la jeunesse (LPJ) et la Loi sur les jeunes contrevenants (LJC), aujourd'hui rebaptisée la Loi sur le système de justice pénale pour les adolescents (LSPJA). 
temps, une analyse transversale des treize récits d'expérience a été menée. Celle-ci a permis de repérer les thèmes récurrents abordés par les participantes, qu'ils donnent lieu à des propos convergents ou divergents sur les aspects à l'étude. Ainsi, les entretiens ont été comparés entre eux et mis en relation afin d'obtenir une vue d'ensemble du matériel et de relever les éléments de ressemblance et de dissemblance qui ressortent de l'expérience vécue par les jeunes filles en lien avec les gangs de rue.

\section{Différentes formes de victimisation reliées à l'affiliation des filles aux gangs}

\section{L'agression physique}

Nos données confirment que les agressions physiques commises à l'endroit des filles peuvent débuter dès leurs premiers contacts avec le gang, au moment de l'initiation. Cette pratique ne paraît toutefois pas très répandue au sein des gangs auxquels les adolescentes interrogées étaient affiliées, une seule d'entre elles affirmant avoir été initiée, l'initiation consacrant son entrée dans le gang. Comme elle aspirait à faire partie du gang au même titre qu'un garçon, Marie-Pierre ${ }^{5}$ a dû vivre l'initiation réservée aux membres masculins, c'est-à-dire être battue par les garçons du gang pendant une période de temps fixée à l'avance:

Alors j'ai passé le test. Ils étaient vingt-quatre et c'étaient des gars assez corpulents, ils n'étaient pas tous des tout maigres. Les tout maigres frappaient à coups de ceinture, et les plus corpulents frappaient à coups de poings ou à coups de claques. Je vais te dire quelque chose: des pinces et des «kicks» à plein élan, des fois, ça fait pas du bien. Alors j'ai passé le test mais, après vingt minutes, j'ai commencé à perdre le cap un peu. C'est sûr que tu te ramasses avec des bleus, tu saignes, tu te tapes trois ou quatre coups de pieds et à un moment donné, ça coupe [Marie-Pierre, 24 ans].

Les propos des autres interviewées laissent entendre qu'elles n'ont pas été elles-mêmes initiées. Elles racontent plutôt avoir eu connaissance que des filles auraient vécu une initiation, généralement à connotation sexuelle. Le type d'initiation subie par Marie-Pierre représenterait donc davantage l'exception que la règle.

5. Tous les noms des jeunes filles dont les propos sont cités ont été changés afin de préserver leur anonymat. 
Nos données confirment aussi que la manifestation de gestes de violence physique à l'endroit des jeunes filles affiliées à un gang ne se limite pas au moment de l'initiation, mais peut perdurer aussi longtemps que s'étend la période de fréquentation du gang, comme en témoignent les jeunes filles rencontrées. Ainsi, la presque totalité d'entre elles ont confié avoir été victimes d'abus physiques commis par leurs acolytes masculins. C'est notamment le cas de Clara, qui affirme avoir été frappée et battue à plusieurs reprises :

J'en ai mangé des volées par eux autres [les membres du gang]. Moi, j'étais soûle bien raide alors je ne m'en rendais pas compte et c'est le lendemain que tout le monde me racontait ça. Le lendemain, c'était rendu que j'avais de la misère à me lever du lit, j'étais pleine de bleus partout [Clara, 15 ans].

Les agressions commises à l'endroit des jeunes filles affiliées au gang se produiraient ainsi tant et aussi longtemps qu'elles fréquentent ce milieu, et même au-delà de cette période, comme nous le verrons. Et bien que plus visibles, les abus physiques ne constituent que l'une des formes de victimisation subies par les adolescentes pendant la période où elles font l'expérience des gangs de rue. Leur témoignage met en effet en lumière qu'elles peuvent également être victimes d'autres types de victimisation, plus subtils, visant cette fois à contrôler leur conduite.

\section{Le contrôle et la violence amoureuse}

Le contrôle s'exerce sur les filles de la bande de deux façons distinctes: par l'amoureux qui fait partie du gang, et par l'ensemble des membres de celuici. Selon les propos des adolescentes, il arriverait fréquemment que la jalousie et la possessivité teintent les relations amoureuses qu'elles vivent avec un membre du gang, ce qu'illustrent bien les propos de Cassandre:

Il est jaloux, possessif au bout et c'est grave. Moi j'aime ça aller chez mes amis, et c'est ses amis à lui aussi, mais il aime pas ça. C'est: «Va travailler, rentre à la maison, fais ci, fais ça». Et à un moment donné, on était en appartement ensemble et là j'étais contrôlée plus que tout au monde. C'était un contrôle que j'ai jamais eu de ma vie, c'était pire qu'en centre de réadaptation. J'étais tellement contrôlée et chronométrée, minute par minute... [Cassandre, 17 ans].

Signes des temps modernes, certains moyens technologiques seraient utilisés à cette fin: notamment le télé-avertisseur et le téléphone cellulaire. Ces appareils permettent en effet à l'amoureux, sous couvert de lui offrir un présent, de joindre sa compagne à tout moment et ainsi de savoir ce 
qu'elle fait, où elle se trouve et de s'assurer qu'elle est toujours disponible en cas de besoin:

Elles [parlant de certaines filles fréquentant les gangs] ont tout le temps une pagette $^{6}$ sur elles ou un cellulaire et quand les gars ont besoin d'elles, ils les «pagent» ou ils leur téléphonent. La plupart des filles ont toutes des pagettes [Yanie, 14 ans].

Les récits que font les adolescentes interviewées signalent à quel point les notions de jalousie, de possessivité, de contrôle et d'obéissance occupent une place importante dans les relations amoureuses qui se déroulent dans le cadre d'un gang. Ce constat a également été fait par d'autres chercheurs, qui soutiennent que les garçons membres de bandes ont tendance à considérer les filles comme une forme de propriété, ce qui les amène à vouloir contrôler leurs faits et gestes (Harris, 1988; Covey et al., 1997).

La violence amoureuse ne s'exerce toutefois pas que par le biais indirect du contrôle. En effet, trois des jeunes filles interviewées rapportent s'être vues empoigner par le bras, poussées ou encore menacées verbalement par leur amoureux, qui faisait alors partie du gang qu'elles fréquentaient. C'est ce que relate Helen:

Mon ex, une fois, m'a pogné le bras... il m’a pogné le bras et il m’a dit: «Si tu t'en vas, je vais te frapper»... Il disait: «Si tu t'en vas pas, je vais pas te frapper», et il me pognait le bras et il me faisait mal [Helen, 16 ans].

Certes, on pourrait arguer que la violence et le contrôle exercés par les garçons sur les filles ne se restreignent pas aux gangs de rue. Il est en effet de plus en plus question de violence dans les relations amoureuses des couples adolescents (Cousineau et al., 2003a). Il est donc difficile de relier ce phénomène aux gangs de façon exclusive et il faut plutôt le considérer, ici, comme un problème lié aux jeunes.

Par contre, ce qui serait davantage caractéristique des gangs de rue tient au fait qu'au sein de ceux-ci, le contrôle et la surveillance des jeunes filles qui y sont affiliées ne seraient pas l'apanage exclusif de l'amoureux. En effet, les autres garçons membres de la bande se montreraient tout aussi dominants, visant à s'assurer que les adolescentes demeurent soumises, dignes de confiance et qu'elles ne fréquentent pas de membres de bandes adverses. Ils se mettent donc à les épier et à gérer leur vie afin qu'elles se plient à leurs règles, voire à leurs désirs, qu'elles demeurent affiliées au gang et qu'elles continuent de contribuer aux profits de celui-ci:

6. Télé-avertisseur. 
Ils surveillent tout le temps, ils sont tout le temps là, ils sont partout. Je vais être à quelque part, ils vont être là. Il y en a tout le temps un quelque part, tout le temps. Ils sont partout et ils sont nulle part en même temps. Ils sont incognitos, ils peuvent tout savoir sur moi. Je peux avoir été dans une fête et le lendemain, ils vont m'appeler, ils vont avoir su. J'ai intérêt à ne pas faire de niaiseries, c'est ça qu'ils me disent [...]. Il faut que je fasse attention à ce que je dis, à ce que je fais. C'est ça qu'ils veulent dire par là, j'ai intérêt à pas faire de niaiseries. Il faut que je leur prouve ma fidélité [Eva, 16 ans].

Le sentiment que le gang est omniprésent est ici bien réel et contribue à mettre les jeunes filles sur leurs gardes, en leur faisant savoir qu'elles n'ont qu'à bien se tenir. De fait, racontent plusieurs adolescentes, lorsque le contrôle échoue et que les filles «trahissent» le gang ou qu'elles agissent de façon contraire à ce qui est attendu d'elles, il arrive qu'elles soient battues par les autres membres du gang. C'est ce qu'a vécu Cassandre, lorsqu'elle a eu un amoureux faisant partie d'une bande ennemie:

Je me suis fait battre parce que j'ai sorti avec un gars de gang adverse [...]. Ça s'accepte pas parce qu'ils disent que je peux les trahir, donner leur adresse et leur numéro de téléphone aux autres gars. Alors ils n'ont pas accepté et ils m'ont frappée pour ça. Et j'ai continué à sortir avec le gars pareil, je suis sortie avec deux mois, mais j'ai eu deux mois de calvaire dans ma vie: ils me laissaient pas vivre. Dès qu'ils me voyaient dans la rue, c'était: «Qu'estce que tu fais?». Ils allaient me frapper, ils allaient me voler des choses. Alors j'ai été obligée de le quitter pour ça [Cassandre, 17 ans].

Ces témoignages révèlent que le contrôle et la violence qui s'y associe souvent ne sont pas seulement exercés par l'amoureux que les filles fréquentent, mais également par l'ensemble des membres du gang. Ce contrôle vise essentiellement à rendre les filles obéissantes et à s'assurer que leurs actions sont toujours posées pour le bien du groupe. Plus concrètement, la surveillance constante des faits et gestes des adolescentes, ainsi que les menaces proférées à leur endroit, permettent aux membres d'exercer leur pouvoir et de s'assurer que les filles se montreront soumises et qu'elles ne les trahiront pas en informant les gangs adverses de leurs activités. La victimisation se fait ici essentiellement psychologique, bien qu'il arrive que des manifestations de violence physique s'y ajoutent lorsque l'emprise du contrôle psychologique se révèle insuffisante ou insatisfaisante. 


\section{L'isolement}

Les gangs de rue cherchent également à isoler les jeunes filles des influences extérieures, sans doute pour exercer un meilleur contrôle sur elles. Les membres du gang s'organisent ainsi pour qu'elles coupent les ponts avec leur famille et avec les amis qu'elles avaient avant de joindre le groupe:

J'avais quasiment pas le droit de parler à personne d'autre, c'était juste eux autres et c'était avec eux autres, personne d'autre [...]. Chaque fois que ma mère appelait, il [1'amoureux] donnait toujours un prétexte pour éviter qu'elle me parle: "Elle est dans la douche», "elle est partie chercher du pain», mais dans le fond, j'étais là. Et j'étais là: «De qui tu parles?». Quand il raccrochait, il disait: "C'est une de mes amies qui a appelé pour parler à son chum et je lui ai dit qu'il était pas là» [Laurie, 15 ans].

Se retrouvant ainsi isolées du monde extérieur, les filles qui cherchent à tisser des liens amoureux se trouvent confinées à vivre ces relations au sein du gang, c'est du moins ce qu'elles ressentent très clairement:

$\mathrm{Tu}$ es une fille, une fille de la gang, tu restes dans la gang et tu t'en vas pas ailleurs. C'est dans leur mentalité: «Tu sors plus avec lui, il va falloir que tu sortes avec un de nous autres» [...]. Tu peux pas choisir en dehors de la gang [Marie-Pierre, 24 ans].

Le gang utiliserait parfois des techniques encore plus drastiques afin d'isoler complètement les jeunes filles de leur entourage. En effet, certaines des interviewées ont raconté que les filles affiliées aux gangs pouvaient être envoyées dans des villes où elles ne connaissent personne, afin d'y faire de la prostitution et de rapporter de l'argent, jusqu'à ce qu'elles commencent à créer des liens et à se débrouiller. On les déplace alors à nouveau :

Ils les amènent à quelque part d'autre et après, elles ne savent pas comment s'en retourner, elles connaissent personne là-bas [...]. Elles ne parlent pas anglais. [...] C'est la meilleure solution pour eux autres parce que la fille ne peut pas retourner, elle peut rien faire. Et quand la fille commence à se débrouiller toute seule, ils n'aiment pas ça [...]. Quand ils sont ici et que la fille est capable de se débrouiller, ils vont partir à quelque part d'autre avec elle, où elle ne connaît personne [Marjorie, 16 ans].

Ainsi, les jeunes filles affiliées à un gang de rue se trouvent contraintes à ne fréquenter que les membres du gang et se voient coupées des relations qu'elles avaient avec ceux et celles qui n'en font pas partie. Interrogées à ce sujet, seulement deux des interviewées ont indiqué qu'elles continuaient de fréquenter des amis à l'extérieur du gang. Quant aux autres, les anciens 
amis ont graduellement cessé de les contacter et de les voir, ce qui les rend encore plus dépendantes du gang, puisque les membres de celui-ci constituent désormais leurs seuls amis, comme le relate Laurie:

Au moins si j'avais eu d'autre monde, peut-être que ça m'aurait aidée mais j'avais vraiment plus personne. Mes amis qui étaient corrects voulaient plus me parler à cause de ça, et à cause de plein d'affaires [Laurie, 15 ans].

$\mathrm{Du}$ fait de cet isolement plus ou moins complet, les filles qui entreprennent de quitter le gang vivent de grandes difficultés, la plus importante étant qu'elles se retrouvent seules avec un réseau social à rebâtir complètement. Finalement, l'isolement permet à la bande d'exercer un meilleur contrôle sur les jeunes filles en s'assurant qu'elles ne subissent aucune influence extérieure.

\section{La victimisation sexuelle}

L'analyse des entrevues a certainement révélé que la victimisation sexuelle est bien présente dans le milieu des gangs de rue, les filles devant se prêter à diverses demandes reliées à leur sexualité, comme l'avaient révélé avant nous Molidor (1996), de même que Joe Laidler et Hunt (1997) notamment. D'abord, les adolescentes peuvent faire l'objet d'un certain trafic qui se passe entre les membres du gang, c'est-à-dire que ceux-ci s'échangent les faveurs sexuelles des filles, qui doivent alors avoir des relations sexuelles sur commande avec un ou plusieurs garçons:

C'était comme un réseau d'escortes, mais plus entre eux autres tu sais, du monde qu'ils connaissaient. C'était pas des gros dealers de femmes, c'était plutôt "gang-bangs», initiation de filles, c'était plus rentable pour eux autres... Ça se passait les filles [Marie-Pierre, 24 ans].

Et c'était ça: «Lui c'est mon meilleur ami et là ce soir ça lui tente alors va donc dans la chambre avec». Moi j'ai jamais été... Je suis une fille bien gênée sur ce point-là, j'ai même de la misère à me déshabiller toute nue devant mon propre chum. C'était vraiment là: «Tu y vas» et là, je disais non et c'était: «Tu veux-tu y aller ou tu veux une claque sur la gueule?» [Laurie, 15 ans].

Les adolescentes qui joignent un gang de rue peuvent également être conduites à pratiquer des métiers liés à la sexualité: danse, escorte ou prostitution, pour satisfaire les besoins financiers du gang (Campbell, 1984; Hamel et al., 1998). La façon d'amener les jeunes filles vers ce type d'occupation peut varier, allant du consentement libre et volontaire à la coercition, en passant par la persuasion. Parmi les jeunes filles interrogées, 
plusieurs ont en effet confié avoir pratiqué les métiers du sexe au profit du gang, et ce, de manière plus ou moins intentionnelle, en répondant aux pressions des membres masculins qui les ont convaincues qu'elles pourraient ainsi faire de l'argent, bien qu'elles n'en aient jamais vu la couleur. Mais si certaines affirment avoir choisi ou accepté de danser et de se prostituer pour le gang, d'autres témoignent plutôt du fait qu'elles se sont vues dans l'obligation de pratiquer de telles activités sous prétexte qu'elles auraient bénéficié des largesses du gang, contractant du même coup une dette envers celui-ci. Pour ces dernières, un refus signifiait être battues, ou du moins menacées de l'être:

Ils [les membres du gang] te donnent de la drogue, ils t'embarquent dans une drogue forte que tu vas continuer à prendre, et tu vas être accro là-dessus. Là, tu vas vouloir leur en demander, ils vont être fins, ils vont tout te donner. Eux autres, ils donnent tout mais après ils disent: «Paye». Là tu as un choix, faire de la prostitution. Là tu fais de la prostitution [...]. C'est juste une question de peur .... [Nancy, 15 ans].

Le témoignage de Nancy rejoint celui d'autres jeunes filles qui expliquent que c'est souvent la peur qui amène à s'engager dans de telles activités. Toutefois, contrairement à ces jeunes filles qui se disent conscientes de ce qui leur arrive, certaines dévoilent des situations où la prostitution serait pratiquée en quelque sorte à l'insu des principales impliquées:

Ils vont demander à une fille d'amener une amie ou des amies. Là, il va aller dans la chambre en lui disant qu'il a des affaires à lui montrer ou il va parler de quelque chose qui va intéresser la fille. Là, il va dans la chambre avec elle et il couche avec, et il y en a un autre qui va rentrer. Là, il trouve une raison pour sortir et un autre va rentrer. Et des fois, quand ça va plus loin, il y a d'autres personnes qui vont rentrer que tu connais pas, des vieux. Alors là le gars est payé, mais la fille le sait pas. C'est comme si elle était en train de se prostituer, mais elle le sait pas [Marjorie, 16 ans].

C'est un fait connu que l'industrie du sexe est assez importante au sein de certains gangs de rue. Les filles qui la font fonctionner, nous venons de le voir, peuvent le faire de façon libre et volontaire, mais elles peuvent également y être forcées ou le faire sans même le savoir. Peut-on alors parler d'exploitation des adolescentes par les gangs? Clara, qui s'est prostituée pour une bande, dirait oui:

Il profitait de moi parce que j'étais en fugue, j'étais tout le temps gelée, j'avais besoin d'argent alors, dans le fond, il profitait de moi. C'est là que je m'en rends compte que je suis mieux en centre de réadaptation que dans la rue [Clara, 15 ans]. 
Nous ne pouvons nier le fait que certains groupes organisés utilisent les adolescentes afin de tirer profit de leur corps, mais nous devons aussi reconnaître que tous les gangs de rue n'en font pas commerce. Toutefois, étant donné les conséquences que peuvent avoir de telles expériences sur la vie des jeunes filles qui les vivent, cet aspect revêt une importance particulière.

Outre le «trafic» de filles pratiqué par les membres du gang et les métiers reliés à la sexualité, les adolescentes affiliées à une bande peuvent également être victimes d'agressions sexuelles. D'ailleurs, plus de la moitié des jeunes filles interviewées ont confié avoir été victimes d'attouchements non désirés et/ou d'agressions sexuelles pendant la période où elles ont été associées à un gang. C'est notamment le cas de Helen:

Il m'a invitée et il m'a amenée dans sa chambre, et après ça il y a plein de gars qui sont venus un par un dans la chambre. Et moi, je comprenais pas c'était quoi et à la fin, j'ai dit au gars: «Je veux m'en aller» et il me laissait pas partir, il m'a embarrée dans la chambre. Et quand il a ouvert la porte, j'ai vu qu'il y avait une file d'attente. Je comprenais rien et j'ai dit au gars: «Laissemoi partir», et il me disait: «Non, je te laisse pas partir, tu restes là» [...]. Il y en a cinq qui m'ont passée dessus et c'était comme vers minuit, une heure du matin, ils m'ont amenée dans un coin du parc, à côté d'un arbre et ils m'ont tous violée [Helen, 16 ans].

À la lumière des récits faits par les adolescentes que nous avons rencontrées, il convient de conclure, à l'instar de Huff (1997), que les agressions sexuelles de toutes sortes constituent l'un des aspects navrants de l'expérience des filles dans le gang.

\section{La peur et les menaces}

Parmi les motifs incitant les adolescentes à poursuivre leur affiliation au gang se trouve la peur que les menaces proférées par les membres du groupe ne soient mises à exécution. Bien que celles-ci ne se concrétisent que rarement (Hamel et al., 1998), la peur semble être suffisamment grande pour retarder le moment où elles en viennent à quitter le gang, d'autant plus qu'elles ont souvent été témoins et victimes de la violence exercée par ses membres. C'est ce que racontent Laurie et Helen, celleci évoquant la notion de courage:

Souvent, j'ai voulu m'en aller mais il [1'amoureux] me retenait toujours... Premièrement, j'avais peur qu'après, c'est du monde fou, $j$ 'avais peur qu'ils viennent mettre une bombe chez nous. Tu sais pas ce monde-là jusqu'où ils 
peuvent aller [...]. J'avais peur, je voyais quels genres de problèmes ils faisaient au monde [...]. Je m'imaginais plein d'affaires, je voulais vraiment pas qu'il arrive rien ni à moi, ni à ma famille, parce qu'ils avaient rien à voir là-dedans. Alors, j'avais bien peur. Quand je suis entrée en centre de réadaptation, ils ont vraiment couru après moi, jusqu'à temps qu'ils appellent chez nous et que ma mère dise: "C'est assez» [Laurie, 15 ans].

Quand j'ai été obligée de les laisser tomber à cause du centre de réadaptation, j'avais de la misère parce qu' ils voulaient que je retourne, ils appelaient chez nous et ils me menaçaient [...]. Il faut que tu aies beaucoup de courage pour te sortir de là parce que c'est pas facile de sortir de là du jour au lendemain, c'est très difficile. Et des fois, ils peuvent menacer de te tuer, ou ils te voient dans la rue et ils vont essayer de te violer, ils vont essayer de tout faire pour prendre leur revanche [Helen, 16 ans].

Ces propos témoignent du fait que la victimisation vécue par les jeunes filles associées à un gang de rue peut se poursuivre jusqu'à ce qu'elles le quittent, et parfois même au-delà de ce départ, ce qui constitue sans nul doute un obstacle au processus de désaffiliation.

\section{En conclusion...}

Le récit d'expérience que font les jeunes filles rencontrées dans le cadre de cette recherche révèle que la plupart d'entre elles ont subi des abus physiques et sexuels de toutes sortes au sein de leur propre gang et qu'elles ont vécu l'isolement et le contrôle exercés par les membres masculins du groupe. Contrairement à ce que l'on pourrait croire, les menaces de victimisation proviendraient ainsi non seulement des bandes adverses, comme d'autres auteurs l'ont observé, mais aussi, et même surtout, du gang d'appartenance. En effet, on pourrait être tenté de penser que les gangs ennemis contribuent à augmenter le nombre d'abus physiques vécus par les jeunes filles mais, de façon surprenante, une seule d'entre elles parle des blessures qu'elle a subies lors de bagarres avec les bandes rivales comme faisant partie des expériences de victimisation qu'elle a vécues.

Ce constat nous conduit à aborder le thème de la lecture différentielle qui peut être faite par les chercheurs et par les jeunes filles elles-mêmes concernant la victimisation subie en lien avec l'expérience vécue auprès des gangs de rue et de leurs membres. En analysant les propos des interviewées concernant la victimisation vécue pendant leur affiliation à un gang, nous constatons une certaine distance entre ce que nous considé- 
rons comme des éléments victimisants et ce que les jeunes filles estiment être de la victimisation. La perception du chercheur differe ici, à maints égards, de celle qu'en ont les adolescentes qui ont fait l'expérience des gangs. Par exemple, lorsqu'on leur demande si elles ont le sentiment d'avoir été l'objet d'abus physiques ou sexuels, les jeunes filles interrogées n'associent pas le contrôle physique qu'elles ont subi, ni l'isolement qui leur a souvent été imposé par les membres du gang, à des situations d'abus. Elles ne paraissent pas considérer que le fait de se voir imposer une forme de contrôle relativement intense, voire excessif, constitue une forme de victimisation et d'abus. Peut-être est-ce parce qu'elles estiment que ce contrôle fait partie de l'univers du gang, qu'il est nécessaire à la survie de ce dernier et qu'il est, par conséquent, normal de se voir imposer une telle surveillance. Cet aspect ferait donc partie des «risques du métier».

Molidor (1996) soutient qu'il est justifié de percevoir les filles affiliées à un gang comme des victimes. Plusieurs ont vécu de la violence physique et sexuelle depuis leur enfance, elles ont été les victimes de conditions économiques difficiles et de pauvreté. Cet auteur rappelle aussi qu'elles ont subi de la violence physique et sexuelle associée aux activités du gang. Toutefois, il estime qu'il est inadéquat de percevoir ces jeunes filles uniquement comme des victimes, puisque leur rôle évolue peu à peu et qu'elles sont aujourd'hui responsables de délits sérieux, se faisant agresseurs à leur tour. Covey et al. (1997) ajoutent que le fait de réduire la participation des filles dans les gangs de rue à la victimisation qu'elles y subissent serait réducteur et dresserait un portrait partiel de ce qu'elles vivent au sein de tels regroupements, ce qui est selon nous tout à fait juste. Il était toutefois impossible, dans le cadre restreint de cet article, d'aborder toutes les facettes de l'expérience des adolescentes affiliées aux gangs de rue.

Il nous paraissait important de mettre spécialement l'accent sur la dimension de la victimisation qui se révèle un aspect prégnant de l'expérience que vivent les jeunes filles affiliées aux gangs du fait même de leur affiliation aux groupes en question. Les résultats obtenus à cet égard nous amènent à exprimer des craintes quant à l'expérience vécue par les adolescentes qui font l'expérience des gangs de rue. Elles y vivent des événements parfois dramatiques, susceptibles de les marquer pour la vie ou, du moins, d'influencer définitivement leur cheminement, ce dont les intervenants qui œuvrent auprès de ces jeunes filles doivent nécessairement tenir compte. Il n'est toutefois pas toujours aisé d'identifier ces expériences de victimisation étant donné les nombreuses hésitations des jeunes filles à aborder le sujet et la tendance qu'elles montrent à l'expri- 
mer en parlant plutôt des autres. Il est à souhaiter que l'éclairage qu'apporte notre étude à cette facette de l'expérience des jeunes filles au sein des gangs soit de nature à susciter une sensibilité nouvelle permettant une intervention qui tienne compte de cette réalité.

\section{Références}

Angers, M. (1992). Initiation pratique à la métbodologie des sciences bumaines. Montréal: Éditions de la Chenelière inc.

Campbell, A. (1984). The girls in the gang. Oxford: Basil Blackwell.

Chesney-Lind, M., Shelden, G.R., \& Joe, K.A. (1996). Girls, delinquency and gang membership. In C.R. Huff (ed.), Gangs in America (185-204). Thousand Oaks: Sage Publications, $2^{\mathrm{e}}$ edition.

Cohen, A.K. (1955). Delinquent boys: the culture of the gang. New York: Free Press.

Cousineau, M.-M., Gravel, S., Lavergne, C., \& Wemmers, J. (2003a). Des victimes et des victimisations. In M. Le Blanc, M. Ouimet \& D. Szabo (eds), Criminologie empirique (193-241). Montréal: Presses de l'Université de Montréal, $3^{\mathrm{e}}$ édition.

Cousineau, M.-M., Hamel, S., \& Desmarais, A. (2003b). Tournée provinciale pour l'établissement d'un portrait du phénomène des gangs au Québec: buit synthèses régionales et une synthèse globale. Montréal: Comité organisateur du Colloque pour la création d'un réseau québécois permanent d'échange sur les jeunes et les gangs de rue.

Covey, H.C., Menard, S.W., \& Franzese, R.J. (1997). Juvenile gangs. Springfield: Charles C. Thomas, $2^{\mathrm{e}}$ édition.

Curry, G.D. (1998). Female gang involvement. Journal of Research in Crime and Delinquency, 35 (1), 100-118.

Esbensen, F.A., \& Deschenes, E.P. (1998). A multisite examination of youth gang membership: does the gender matter? Criminology, 36 (4), 799-827.

Esbensen, F.A., Deschenes, E.P., \& Winfree, L.T. (1999). Differences between gang girls and gang boys: results from a multisite survey. Youth and Society, 31 (1), 27-53.

Fagan, J. (1996). Gangs, drugs, and neighborhood change. In C.R. Huff (ed.), Gangs in America (39-74). Thousand Oaks: Sage Publications, $2^{\mathrm{e}}$ édition.

Fréchette, M., \& Le Blanc, M. (1997). Délinquance et délinquants. Boucherville: Gaëtan Morin.

Fournier, M. (sous la direction de M.-M. Cousineau) (2003). Jeunes filles affiliées aux gangs de rue, à Montréal: cheminements et expériences. Les Cahiers de recherche criminologique, cahier no. 39. Montréal: Centre international de criminologie comparée.

Hamel, S., Fredette, C., Blais, M.-F., \& Bertot, J. (en collaboration avec M.-M. Cousineau) (1998). Jeunesse et gangs de rue (phase II): résultats de la rechercheterrain et proposition d'un plan stratégique quinquennal. Montréal: Institut de recherche pour le développement social des jeunes. 
Harris, M.G. (1988). Cholas: latino girls and gangs. New York: AMS.

Huff, C.R. (1997). The criminal behavior of gang members in Obio, Colorado and Florida. Communication présentée à la rencontre annuelle de la Western Society of Criminology, Honolulu.

Joe, K.A., \& Chesney-Lind, M. (1995). Just every mother's angel: an analysis of gender and ethnic variations in youth gang membership. Gender and Society, 9 (4), 408-431.

Joe Laidler, K.A., \& Hunt, G. (1997). Violence and social organization in female gangs. Social Justice, 24 (4), 148-169.

Klein, M.W. (1995). The American street gang. Its nature, prevalence, and control. New York: Oxford University Press.

Lauritsen, J.L., Sampson, R.J., \& Laub, J.H. (1991). The link between offending and victimization among adolescents. Criminology, 29 (2), 265-292.

Miller, J. (1998). Gender and victimization risk among young women in gangs. Journal of Research in Crime and Delinquency, 35 (4), 429-453.

Molidor, C.E. (1996). Female gang members : a profile of aggression and victimization. Social Work, 41 (3), 251-257.

Shelden, R.G., Tracy, S.K., \& Brown, W.B. (1996). Girls and gangs : a review of recent research. Juvenile and Family Court Journal, 47 (1), 21-39.

Trasher, F.M. (1927). The gang: a study of 1,318 gangs in Chicago. Chicago: University of Chicago Press. 